\title{
New species, new synonymies and a new record of the genus Cryptogonus Mulsant, 1850 (Coleoptera, Coccinellidae) from China
}

\author{
Lizhi Huo ${ }^{1}$, Wenjing $\mathrm{Li}^{1}$, Xiaosheng Chen ${ }^{1}$, Shunxiang Ren ${ }^{1}$, Xingmin Wang ${ }^{1}$ \\ 1 Engineering Research Center of Biological Control, Ministry of Education; College of Agriculture, South China Agricultural University, Guangzhou \\ 510642, China
}

http://zoobank.org/F4F3EB99-1A69-4FCE-865A-96F22988F346

Corresponding author: Shunxiang Ren (shxren@scau.edu.cn); Xingmin Wang (wangxmcn@scau.edu.cn)

Received 6 May 2015

Accepted 3 August 2015

Published 11 August 2015

Academic editor:

Dominique Zimmermann

\section{Key Words}

\section{Cryptogonus}

new species

new synonymy

new record

China

\begin{abstract}
Three new species of the genus Cryptogonus Mulsant, 1850 from China are described and illustrated: C. dulongjiangensis, C. fusiformis and C. reniformis Huo and Ren. Cryptogonus octoguttatus Mader, 1954 and C. kurosawai Sasaji, 1968 are recognized as synonymous with C. schraiki Mader, 1933. Cryptogonus hingstoni Kapur, 1948 is newly recorded from China. A species list of the genus Cryptogonus is presented.
\end{abstract}

\section{Introduction}

The genus Cryptogonus belongs to Aspidimerini (Coleoptera, Coccinellidae). The Aspidimerini species are widely distributed in South and Southeast Asia. They are natural enemies of coccidae, aphidae, aleyrodidae and have important application value in the control of insect pests.

The genus Cryptogonus was erected by Mulsant (1850) with $C$. orbiculus (Gyllenhal, 1808) as the type species by monotypy. Weise (1900) separated the genera Cryptogonus and Aspidimerus Mulsant, 1850 from Scymnini based on the structure of male genitalia and erected the tribe Aspidimerini with Aspidimerus Mulsant, 1850 as the type genus. Kapur (1948) revised the tribe Aspidimerini and proposed two new genera: Pseudaspidimerus Kapur and Acarinus Kapur, and subdivided the genus Cryptogonus into six species groups based on the shape of prosternal carinae. In Kapur's revision, 19 Cryptogonus species were included, and then during the past several decades
30 new species were attributed to this genus (Mader 1954, Sasaji 1968, Bielawski 1972, 1979, Ghorpade 1974, Pang and Mao 1979, Hoàng 1982, 1985, Cao and Xiao 1984, Canepari 1986, Kuznotsov and Pang Hong 1991, Xiao and Li 1992, Yu 1995, Pang 1998). Among those 30 new species, C. nigritus Pang \& Mao, 1979 and C. montanus Hoàng, 1985 were transferred to Aspidimerus Mulsant, 1850 and Trigonocarinatus Huo \& Ren, 2015, respectively (Kovář 2007, Huo et al. 2015).

During studies on Aspidimerini from the Oriental Region, the genus Aspidimerus from China and Pseudaspidimerus from Laos have been reviewed (Huo et al. 2013, 2014). In the present paper, three new species of the genus Cryptogonus Kapur, 1948 from China are described and illustrated. Cryptogonus octoguttatus Mader, 1954 and C. kurosawai Sasaji, 1968 are recognized as synonymous with C. schraiki Mader, 1933. Cryptogonus hingstoni Kapur, 1948 is newly recorded from China. A species list of the genus Cryptogonus is also presented. 


\section{Material and methods}

All studied materials were deposited in the Department of Entomology, South China Agricultural University (SCAU). Type specimens designated in the current article were deposited in SCAU and the Institute of Zoology (IOZ), Chinese Academy of Science, Beijing.

Measurements were made using an ocular micrometer attached to a stereomicroscope (SteREO Discovery V20, Zeiss) as follows: (TL) total length, from apical margin of clypeus to apex of elytra; (TW) total width, across both elytra at widest part; (TH) total height, through the highest point of elytra to elytral outer margins; (HW) head width, including eyes; (PL) pronotal length, from middle of anterior margin to base of pronotum; (PW) pronotal width at widest part; (EL) elytral length, along suture, from apex to base including scutellum; (EW) elytral width, across both elytra at widest part; (ID) interocular distance, nearest distance between two eyes. Morphological terms follow Ślipiński (2007) and Ślipiński and Tomaszewska (2010) and are applied as in our previous studies on Chinese species of former Scymninae (e.g. Chen et al. 2013, Chen et al. 2014).

External morphology was observed with a stereomicroscope (SteREO Discovery V20, Zeiss). Male and female genitalia were dissected, cleared in $10 \% \mathrm{NaOH}$ solution by boiling for several minutes and observed under a compound microscope, Olympus BX51. Images were photographed with digital cameras (AxioCam HRc and Coolsnap-Procf \& CRI Micro*Color). The software AXIOVISION REL. 4.8 and IMAGE-PRO PLUS 5.1 were used to capture images from both cameras. Images were cleaned up and arranged in plates with ADOBE PHOTOSHOP CS5.

\section{Taxonomy}

\section{Description of new species}

\section{Cryptogonus dulongjiangensis Huo \& Ren, sp. n.}

http://zoobank.org/451E691D-0181-454D-AC81-58830AA25652 Figure 1

Diagnosis. This species can be distinguished from other Cryptogonus species by its long penis, regularly narrowing to pointed apex, penis capsule with both branches very short (Fig. 1e).

Description. TL: $2.37-2.60 \mathrm{~mm}$, TW: $1.86-2.11 \mathrm{~mm}$, TH: 1.21-1.32 mm, TL/TW: 1.23-1.27; PL/PW: 0.510.53; EL/EW: 0.99-1.00, HW/PW: 0.61-0.63; PW/EW: 0.71-0.73. ID/HW: 0.49-0.53.

Body rounded, densely covered with short pubescence, golden on head and pronotum and silver white on elytra (Fig. 1a-c). Head yellow in male and black in female, clypeus dark brown. Pronotum black except a triangular yellow spot at anterior corner in male. Scutellum and elytra black. Ventral side black except legs and abdomen partially yellow to dark brown.
Punctures on frons coarse and dense, 0.3-0.5 diameters apart. Punctures on pronotum and elytra fine and sparse, 1-3 diameters apart. Punctures on metaventrite fine and sparse at middle, 5 diameters apart, coarse and dense on both sides, 0.2 diameters apart.

Male genitalia. Penis long, regularly narrowing to pointed apex (Fig. 1f), penis capsule with both branches very short (Fig. 1e). Tegminal strut as long as tegmen (Fig. 1h). Parameres 2 times length of phallobase with apices sparsely setose. Penis guide, in lateral view, gradually narrowing to pointed apex. In ventral view, subparallel at basal 2/3, narrowing to pointed apex (Fig. 1g).

Female genitalia. Coxites subtriangular (Fig. 1i), with dense, long terminal setae. Spermatheca missing.

Types. Holotype: 1 male, CHINA: Yunnan Prov.: Qinglandan, Maku Village, Dulongjiang, Gongshan County, $27^{\circ} 41.12^{\prime} \mathrm{N}, 98^{\circ} 16.35^{\prime} \mathrm{E}$, ca $1260 \mathrm{~m}, 4-7$. VIII.2010, Wang XM et al. leg. (SCAU). Paratypes (2): CHINA: Yunnan Prov.: 1 male, Maku Village, Dulongjiang, Gongshan County, $27^{\circ} 40.57^{\prime} \mathrm{N}, 98^{\circ} 18.15^{\prime} \mathrm{E}$, ca 1600 m, 1.VIII.2010, Wang XM et al. leg; 1 female, Bapo Village, Dulongjiang, Gongshan County, $27^{\circ} 44.08^{\prime} \mathrm{N}$, $98^{\circ} 20.59^{\prime} \mathrm{E}$, ca $1400 \mathrm{~m}, 28$.VII.2010, Wang XM et al. leg. (SCAU).

Distribution. China (Yunnan).

Etymology. The specific epithet refers to the type locality, Dulongjiang, Yunnan.

\section{Cryptogonus fusiformis Huo \& Ren, sp. n.}

http://zoobank.org/BAD66DB0-5B95-4DF4-949E-EEA46932F585 Figure 2

Diagnosis. This species can be distinguished from other Cryptogonus species by its fusiform penis guide in ventral view (Fig. 2f).

Description. TL: $2.68 \mathrm{~mm}$, TW: $2.14 \mathrm{~mm}, \mathrm{TH}: 1.36$ mm, TL/TW: 1.25; PL/PW: 0.51; EL/EW: 1.00, HW/ PW: 0.58; PW/EW: 0.72. ID/HW: 0.50 .

Body rounded, densely covered with short, silver white pubescence (Fig. 2a-b). Base of head yellow, anterior part black and clypeus dark brown. Dorsum entirely black. Ventral side black except legs and abdomen partially reddish brown.

Punctures on frons dense and coarse, 0.5-1.0 diameters apart. Punctures on pronotum and elytra fine and sparse, 2-4 diameters apart. Punctures on metaventrite fine and sparse at middle, 5 diameters apart, coarse and dense on both sides, 0.3 diameters apart.

Male genitalia. Penis long with apex pointed. Penis capsule with longer outer branch and short inner one. Tegminal strut slightly longer than tegmen. Parameres 2 times length of phallobase with apex densely setose (Fig. 2g). Penis guide, in lateral view strongly curved as $\mathrm{S}$-shape, equivalent to parameres, in ventral view gradually broadening toward midlength, narrowing to pointed apex (Fig. 2f).

Female genitalia. Unknown. 


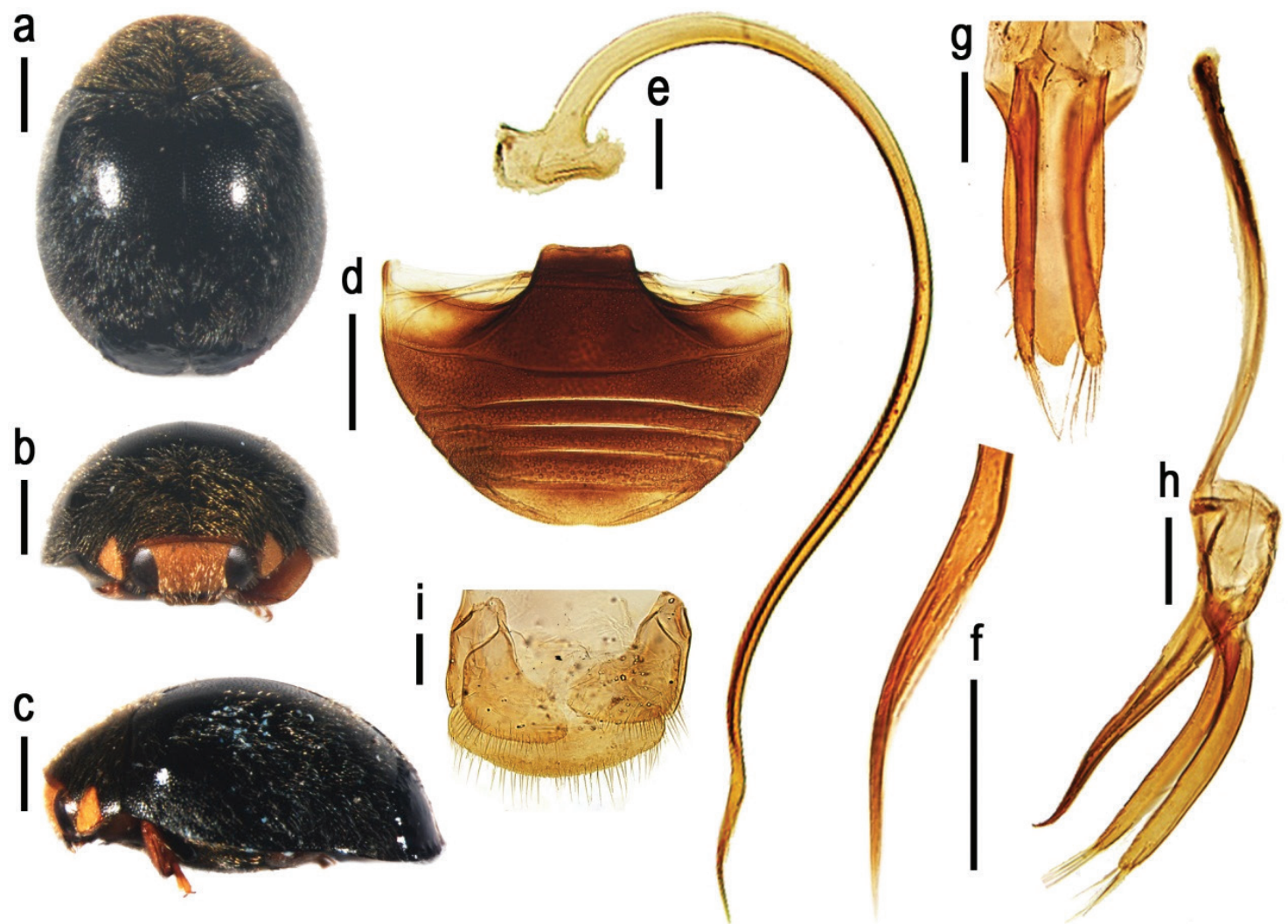

Figure 1. Cryptogonus dulongjiangensis Huo et Ren, sp. n. (a) dorsal view; (b) frontal view; (c) lateral view; (d) abdomen, male; (e) penis; (f) apex of penis; (g) ventral view of tegmen; (h) lateral view of tegmen; (i) coxites. Scale bars: a-d, $0.5 \mathrm{~mm}$; $\mathbf{e}-\mathbf{h}, 0.1 \mathrm{~mm}$.
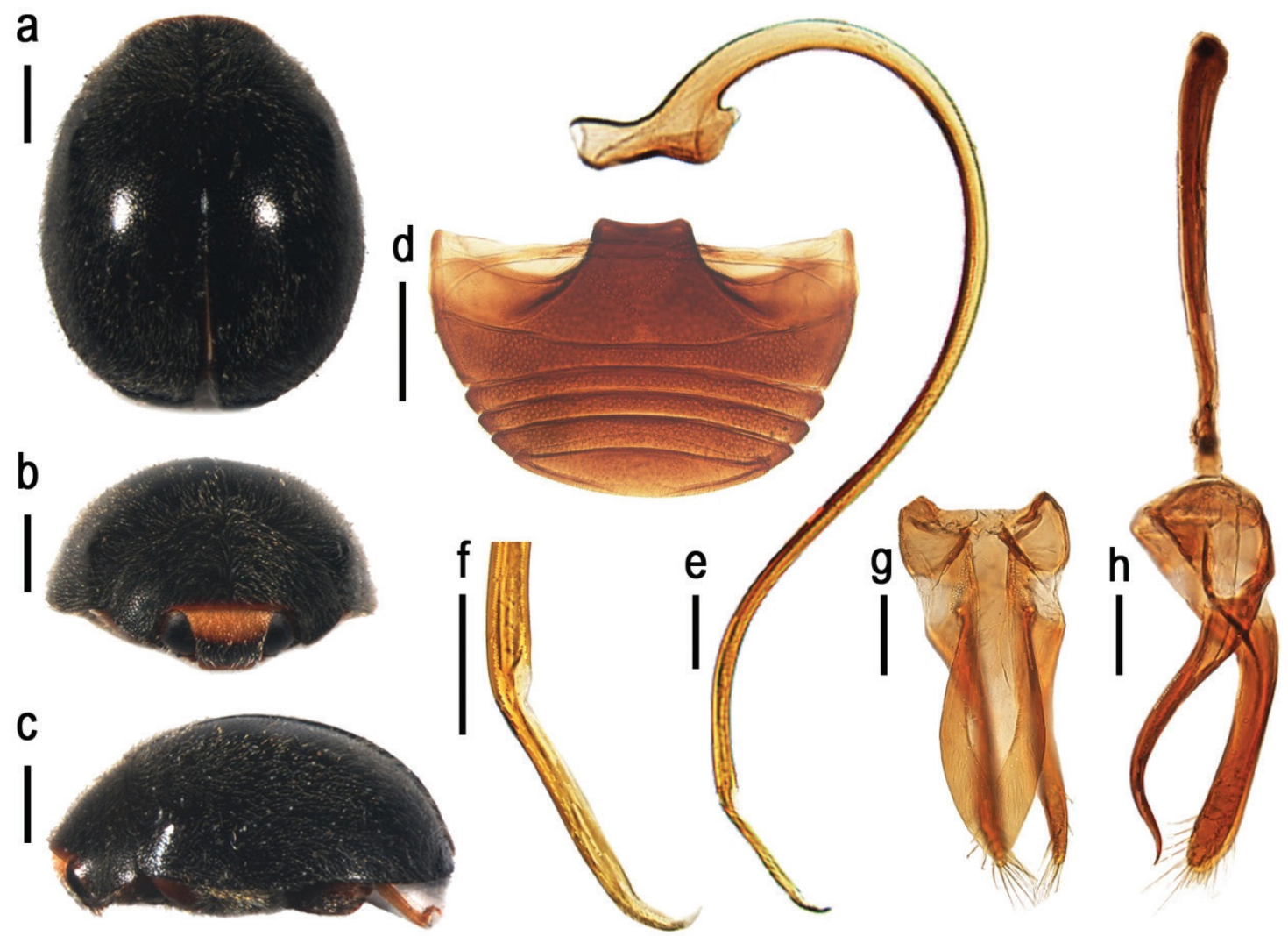

Figure 2. Cryptogonus fusiformis Huo et Ren, sp. n. (a) dorsal view; (b) frontal view; (c) lateral view; (d) abdomen, male; (e) penis; (f) apex of penis; (g) ventral view of tegmen; (h) lateral view of tegmen. Scale bars: a-d, $0.5 \mathrm{~mm}$; $\mathbf{e}-\mathbf{g}, 0.1 \mathrm{~mm}$. 
Types. Holotype: 1 male, CHINA: Yunnan Prov.: Mengdui Town, Zhenkang County, $23^{\circ} 54.16^{\prime} \mathrm{N}$, $98^{\circ} 54.02^{\prime} \mathrm{E}$, ca $1400 \mathrm{~m}, 18 . \mathrm{V} .2008$, Wang XM et al. leg. (SCAU).

Distribution. China (Yunnan).

Etymology. The specific epithet is a Latin adjective referring to its fusiform penis guide in ventral view.

\section{Cryptogonus reniformis Huo \& Ren, sp. n.}

http://zoobank.org/DE7458AF-F2EE-4020-88E0-37465A5B1E96 Figure 3

Diagnosis. This species can be easily identified by its reniform spot on elytra and trifurcate penis apex (Fig. 3a, h).

Description. TL: $1.90-2.40 \mathrm{~mm}$, TW: $1.52-1.94 \mathrm{~mm}$, TH: $1.00-1.27 \mathrm{~mm}$, TL/TW: $1.24-1.25$; PL/PW: $0.53-$ 0.55; EL/EW: 0.96-1.01, HW/PW: 0.61-0.64; PW/EW: 0.71-0.72. ID/HW: 0.49-0.52.

Body rounded, densely covered with short, silver white pubescence (Fig. 3a-c). Head yellowish in male and black in female, clypeus dark brown. Pronotum black except a triangular yellowish spot at anterior corner in male. Scutellum black. Elytra black with a reniform spot at middle (Fig. 3a). Underside black except legs partially yellowish.

Punctures on frons coarse and dense, 0.5-1.5 diameters apart. Punctures on pronotum and elytra fine and sparse, 2-4 diameters apart. Punctures on metaventrite fine and sparse at middle, 8 diameters apart, coarse and dense on both sides, 0.5 diameters apart.

Male genitalia. Penis long with apex trifurcate (Fig. $3 \mathrm{~h})$. Penis capsule with outer branch bigger than inner one, anterior margin deeply concave (Fig. 3g). Tegminal strut as long as tegmen. Parameres 2 times length of phallobase with apex sparsely setose (Fig. 3j). Penis guide, in lateral view gradually narrowing to pointed apex, a little longer than parameres, in ventral view 3 times as long as wide, slightly broadening to apical $1 / 3$, gradually narrowing to rounded apex (Fig. 3i).

Female genitalia. Coxites reniform with a small projection on the apical end (Fig. 3e). Spermatheca short and strongly arcuate without ramus (Fig. 3f).

Types. Holotype: 1 male, CHINA: Yunnan Prov.: Qinglandan, Maku Village, Dulongjiang, Gongshan County, $27^{\circ} 41.12^{\prime} \mathrm{N}, 98^{\circ} 16.35^{\prime} \mathrm{E}$, ca $1260 \mathrm{~m}, 4-7$. VIII.2010, Wang XM et al. leg. (SCAU). Paratypes (38): CHINA: Yunnan Prov.: 6 males, 14 females (2 males and 2 females in IOZ, 4 males and 12 females in SCAU), with the same data as holotype; 1 male, Kongdang Village, Dulongjiang, Gongshan County, $27^{\circ} 52.18^{\prime} \mathrm{N}, 98^{\circ} 20.24^{\prime} \mathrm{E}$, ca $1600 \mathrm{~m}, 27$. VII.2010, Wang $\mathrm{XM}$ et al. leg. (SCAU); 4 males, Bapo Village, Dulongjiang, Gongshan County, $27^{\circ} 44.09^{\prime} \mathrm{N}, 9^{\circ} 21.02^{\prime} \mathrm{E}$, ca 1400 $\mathrm{m}, 28$.VII.2010, Wang XM et al. leg. (SCAU); 3 males, 3 females, Bapo Village-Maku Village, Dulongjiang, Gongshan County, $27^{\circ} 42.49^{\prime} \mathrm{N}, 98^{\circ} 20.18^{\prime} \mathrm{E}$, ca $1450 \mathrm{~m}$, 29.VII.2010, Wang XM et al. leg. (SCAU); 4 males, 1 female, Maku Village, Dulongjiang, Gongshan County, $27^{\circ} 40.57^{\prime} \mathrm{N}, 98^{\circ} 18.15^{\prime} \mathrm{E}$, ca $1600 \mathrm{~m}$, 1.VIII.2010, Wang $\mathrm{XM}$ et al. leg. (SCAU); Tibet: 2 females, Beibeng Village, Motuo County, ca 850 m, 4.X.2011, Huo LZ et al. leg. (SCAU).

Distribution. China (Tibet, Yunnan).

Etymology. The specific epithet is a Latin adjective referring to its reniform spot on elytra.

\section{New synonymies and new record}

\section{Cryptogonus schraiki Mader, 1933}

Figures 4, 5

Cryptogonus schraiki Mader, 1933: 80; Kapur 1948: 101; Pang and Mao 1979: 69; Kováŕ 2007: 576; Ren et al. 2009: 120.

Cryptogonus octoguttatus Mader, 1954: 129; Pang and Mao 1979: 68; Kováŕ 2007: 576; Ren et al. 2009: 118. Syn. $\mathbf{n}$.

Cryptogonus kurosawai Sasaji, 1968: 11; Yu 1995: 140, 2011: 161; Kovár 2007: 576. Syn. n.

Remarks. Leopold Mader described C. schraiki Mader, 1933 from Sichuan, China with a brief description of elytral coloration (Fig. 4a-i). Kapur (1948) reviewed this species and illustrated its appearance. Later, Mader described C. octoguttatus Mader, 1954 also from Sichuan, China, only with description of elytral coloration. Sasaji (1968) described C. kurosawai Sasaji, 1968 from Taiwan, China. Photographs of the holotype were available on the website of The Digital Museum of Natural \& Science. Pang and Mao (1979) reviewed C. schraiki and C. octoguttatus and illustrated their appearance and male genitalia, but didn't notice the similarities of these two species. We examined these three species from China and found that they are just the same species with different elytral coloration. The elytral coloration is variable, from entirely black to entirely yellowish (Fig. 5a-1). Besides, we found the male genitalia of specimens are slightly different, even in the same coloration. Sometimes penis guide equal to, slightly longer or shorter than parameres. They are considered as individual differences.

Material examined. 205 specimens from China were examined (see the details in supplementary material).

Distribution. China (Anhui, Fujian, Gansu, Guangdong, Guizhou, Hubei, Hunan, Sichuan, Taiwan, Yunnan).

\section{Cryptogonus hingstoni Kapur, 1948}

Figure 6

Cryptogonus hingstoni Kapur, 1948: 103; Kapur 1963: 24; Kovář 2007: 575.

Remarks. Kapur (1948) described this species from Sikkim, India. Two specimens from Tibet, China match the description except coloration variation on elytra. In the 


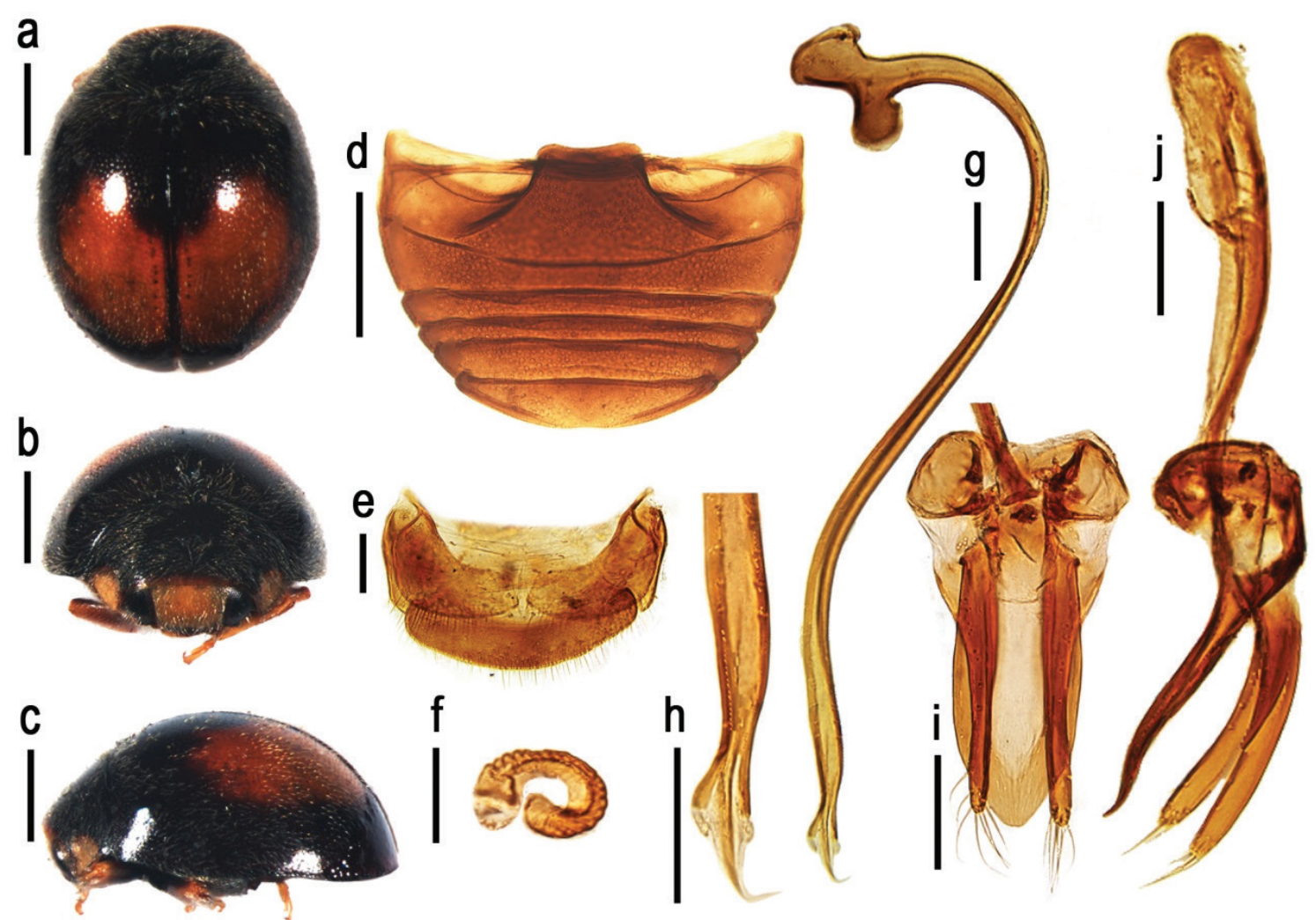

Figure 3. Cryptogonus reniformis Huo \& Ren, sp. n. (a) dorsal view; (b) frontal view; (c) lateral view; (d) abdomen, male; (e) coxites; (f) spermatheca; (g) penis; (h) apex of penis; (i) ventral view of tegmen; (j) lateral view of tegmen. Scale bars: a-d, $0.5 \mathrm{~mm}$; e, $\mathbf{g}-\mathbf{j}, 0.1 \mathrm{~mm} ; \mathbf{f}, 0.05 \mathrm{~mm}$.
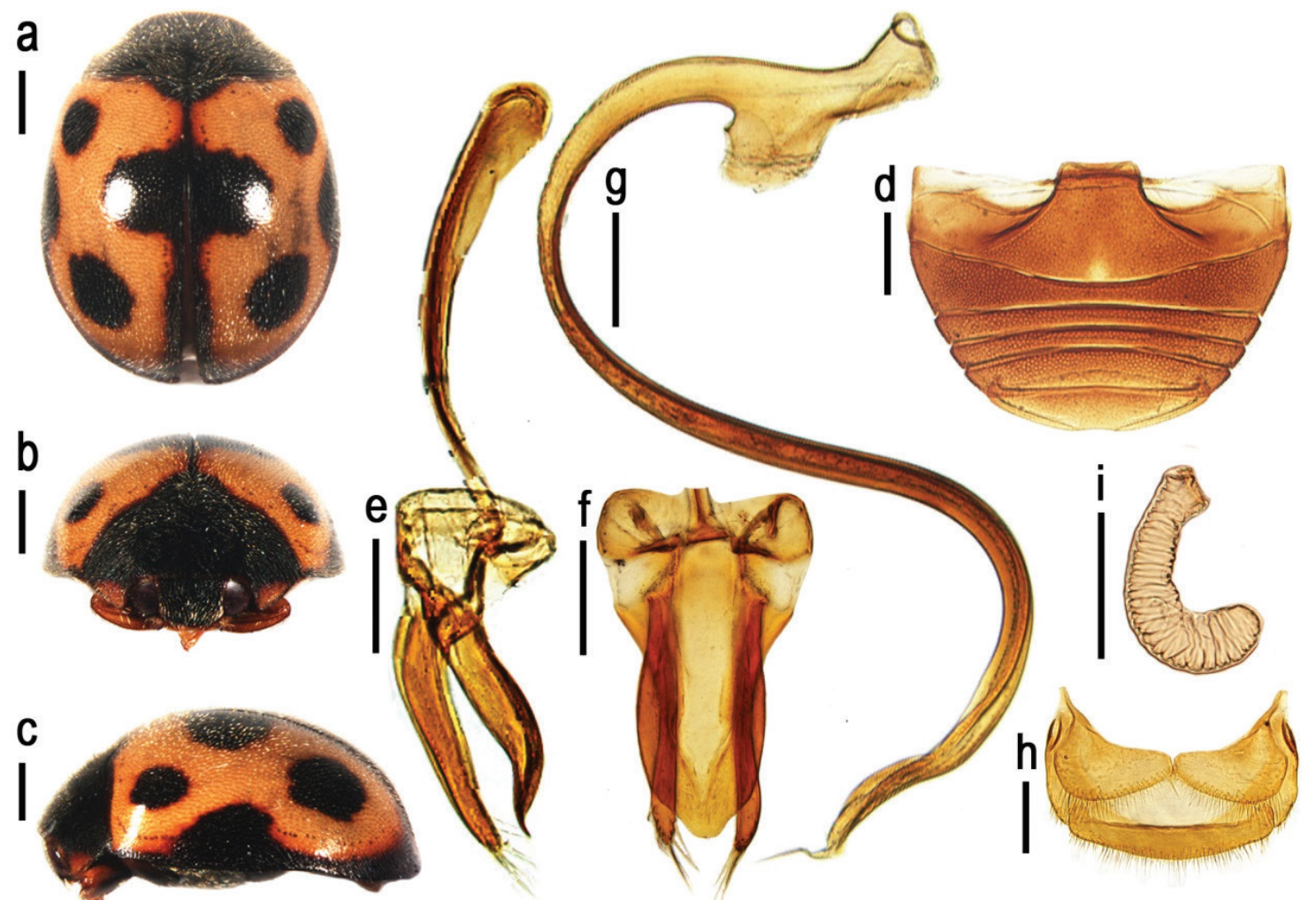

Figure 4. Cryptogonus schraiki Mader, 1933. (a) dorsal view; (b) frontal view; (c) lateral view; (d) abdomen, male; (e) lateral view of tegmen; (f) ventral view of tegmen; (g) penis; (h) coxites; (i) spermatheca. Scale bars: a-d, $0.5 \mathrm{~mm}$; e-h, $0.2 \mathrm{~mm}$; i, $0.1 \mathrm{~mm}$. 

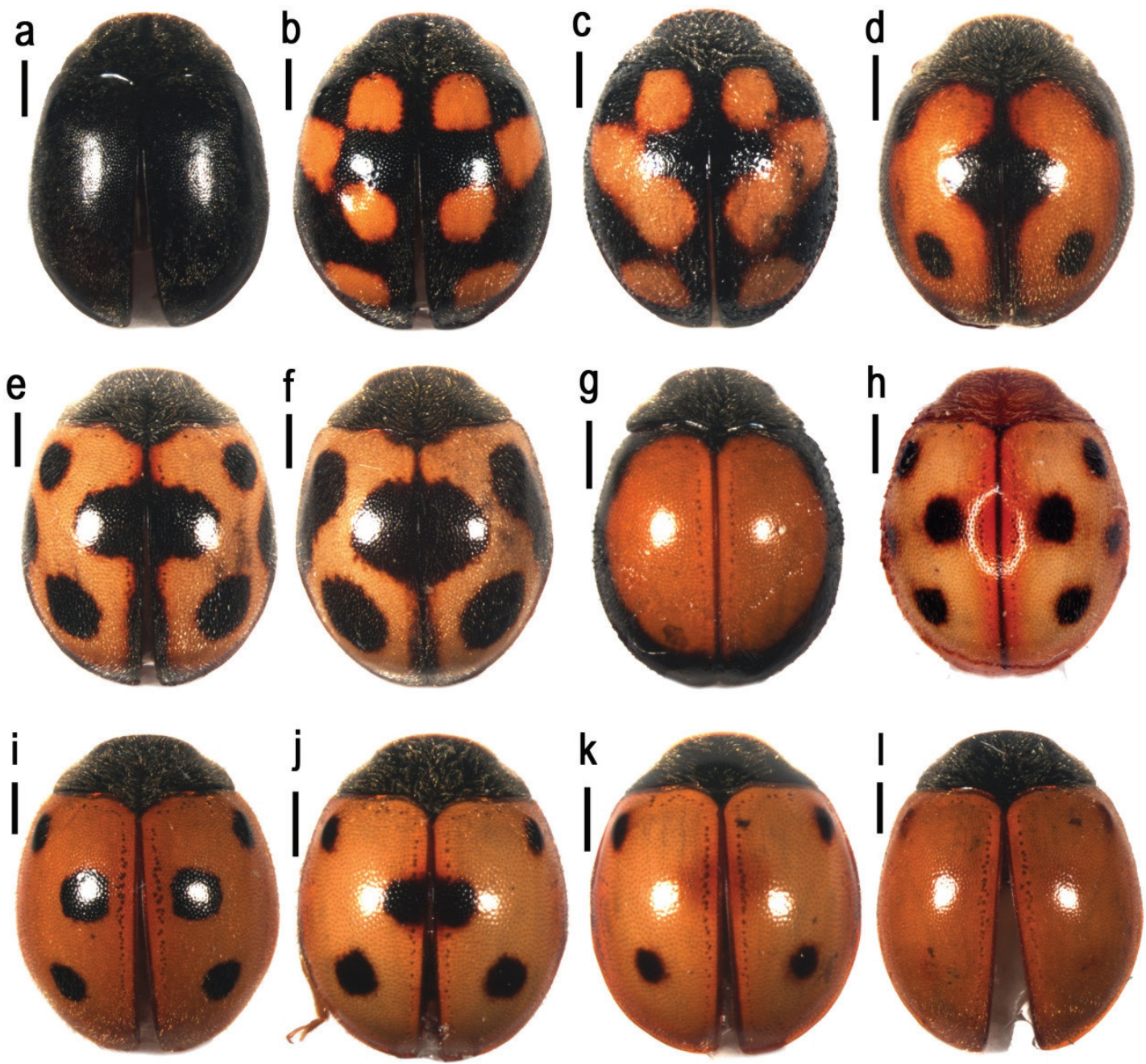

Figure 5. Different elytral coloration of Cryptogonus schraiki Mader, 1933. Scale bars: a-l, 0.5 mm.

original description, the spots on sutural and middle part of the elytron distinctly larger than that on humeral callus, the spot on pronotum is oval. However, in the present specimens we examined, the spots on sutural and middle part are as large as that on humeral callus, the spot on pronotum is triangular (Fig. $6 \mathrm{a}-\mathrm{c}$ ).

Material examined. CHINA: Tibet: 1 male, Bangxin Village, Motuo County, $29^{\circ} 34.58^{\prime} \mathrm{N}, 9^{\circ} 23.60^{\prime} \mathrm{E}$, ca 1840 m, 12.X.2011, Huo LZ et al. leg; 1 female, Beibeng Village-Hanmi Village, Motuo County, $29^{\circ} 14.31^{\prime} \mathrm{N}$, $95^{\circ} 10.58^{\prime} \mathrm{E}$, ca 800-2100 m, 5-8.X.2011, Huo LZ et al. leg.

Distribution. China (Tibet) new distribution, India (Sikkim).

\section{List of the genus Cryptogonus Mulsant}

1. Cryptogonus angusticarinatus Sasaji, 1968

2. Cryptogonus ariasi (Mulsant, 1853)

3. Cryptogonus bilineatus Kapur, 1948

4. Cryptogonus bimaculatus Kapur, 1948

5. Cryptogonus brachylobius Pang, 1998

6. Cryptogonus bryanti Kapur, 1948

7. Cryptogonus complexus Kapur, 1948
8. Cryptogonus deltodirus Kapur, 1948

9. Cryptogonus deltoides Kapur, 1948

10. Cryptogonus downingi Kapur, 1948

11. Cryptogonus dulongjiangensis Huo \& Ren, sp. n.

12. Cryptogonus forficulae Cao \& Xiao, 1984

13. Cryptogonus fractemaculatus Pang, 1998

14. Cryptogonus fulvoterminatus Boheman, 1858

15. Cryptogonus fusiformis Huo \& Ren, sp. n.

16. Cryptogonus guangdongiensis Pang \& Mao, 1979

17. Cryptogonus hainanensis Pang \& Mao, 1979

18. Cryptogonus hanoiensi Hoàng, 1982

19. Cryptogonus himalayensis Kapur, 1948

20. Cryptogonus hingstoni Kapur, 1948

21. Cryptogonus horishanus (Ohta, 1929)

22. Cryptogonus kapuri Ghorpade, 1974: 55

23. Cryptogonus laetus (Weise, 1885)

24. Cryptogonus langchanhensis Hoàng, 1982

25. Cryptogonus lepidus (Weise, 1885)

26. Cryptogonus lijiangiensis Pang \& Mao, 1979

27. Cryptogonus linguilatus Pang, 1998

28. Cryptogonus lobulus Xiao, 1992

29. Cryptogonus loebli Canepari, 1986: 27

30. Cryptogonus nepalensis Bielawski, 1972 

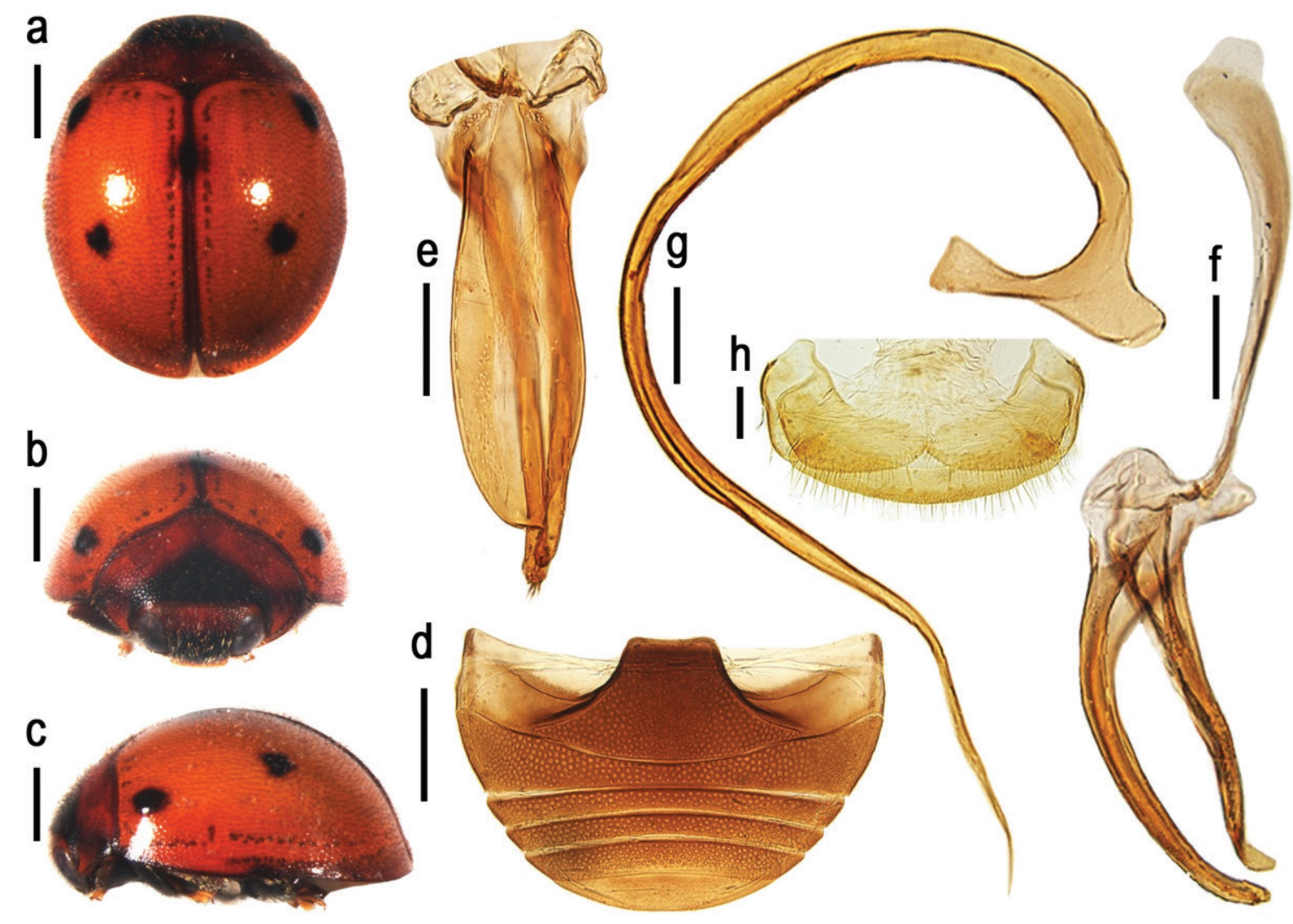

Figure 6. Cryptogonus hingstoni Kapur, 1948. (a) dorsal view; (b) frontal view; (c) lateral view; (d) abdomen, male; (e) ventral view of tegmen; (f) lateral view of tegmen; (g) penis; (h) coxites. Scale bars: a-d, $0.5 \mathrm{~mm}$; $\mathbf{e}-\mathbf{h}, 0.1 \mathrm{~mm}$.

Cryptogonus nepalensis bhutanensis Bielawski, 1979

31. Cryptogonus nitidus Kapur, 1948

32. Cryptogonus ocellatus Hoàng, 1985

33. Cryptogonus ohtai Sasaji, 1968

34. Cryptogonus orbiculus (Gyllenhal, 1808)

35. Cryptogonus parorbiculus Kuznotsov \& Pang Hong, 1991

36. Cryptogonus polytrichus Kuznotsov \& Pang Hong, 1991

37. Cryptogonus postmedialis Kapur, 1948: 95

38. Cryptogonus qianjiangensis Xiao, 1992

39. Cryptogonus quadriguttatus (Weise, 1895)

40. Cryptogonus reniformis Huo \& Ren, sp. n.

41. Cryptogonus robustus $\mathrm{Yu}, 1995$

42. Cryptogonus sagittiformis Pang \& Mao, 1979

43. Cryptogonus schraiki Mader, 1933

44. Cryptogonus trifurcatus Pang \& Mao, 1979

45. Cryptogonus trioblitus (Gorham, 1895)

46. Cryptogonus tristis (Weise, 1910)

47. Cryptogonus wuzhishanus Pang \& Mao, 1979

48. Cryptogonus xiushanensis Xiao, 1993

49. Cryptogonus yunnanensis Cao \& Xiao, 1984

\section{Acknowledgements}

The authors sincerely thank Rashid Azad (SCAU) for improving this manuscript. The research was supported by the Science and Technology Partnership Program, Ministry of Science and Technology of China (KY201402014) and Science and Technology Program of Guangzhou, China (151800033).

\section{References}

Bielawski R (1972) Die Marienkäfer (Coleoptera: Coccinellidae) aus Nepal. Fragmenta Faunistica 18: 283-312. doi: 10.3161/00159301 FF1972.18.16.283

Bielawski R (1979) Ergebnisse der Bhutan-Expedition 1972 des Naturhistorischen Museums in Basal, Coleoptera: Fam. Coccinellidae. Entomologica Basiliensia 4: 83-125.

Boheman CH (1859) Coleoptera. Species novas descripsit. In: Virgin C (Ed.) Kongliga Svenska Fregatten Eugenies Resa omkring jorden under befäl af C.A. Virgin Ahren 1851-1853. Vetenskapliga iakttagelser på H.M. Konung Oscar Den Förstes befallning utgifna af K. Svenska Vetenskaps Akademien, Zoologi III, Inseckter. P.A. Norstedt \& söner, Stockholm, Sweden, 113-218.

Canepari C (1986) On some coccinellids of northern India and Nepal in the Geneva Museum of Natural History (Coleoptera Coccinellidae). Revue Suisse de Zoologie 93(1): 21-36.

Cao CY, Xiao NN (1984) New species of Coccinellidae from Yunnan, China. Entomotaxonomia 6(2-3): 109-125.

Chen XS, Li WJ, Wang XM, Ren SX (2014) A review of the subgenus Neopullus of Scymnus (Coleoptera: Coccinellidae) from China. Annales Zoologici 64(2): 299-326. doi: 10.3161/000345413X672483 
Chen XS, Wang XM, Ren SX (2013) A review of the subgenus Scymnus of Scymnus from China (Coleoptera: Coccinellidae). Annales Zoologici 63(3): 417-499. doi: 10.3161/000345413X672483

Ghorpade KD (1974) Description of a new Cryptogonus Mulsant from Bangalore, Southern India. Oriental Insects 8(1): 55-60. doi: 10.1080/00305316.1974.10434442

Gorham HS (1895) On the Coccinellidae collected by Mr. L. Fea in Burma. Annali del Museo Civicodi Storia Naturale, Genova (2)34: 683-695.

Gyllenhal L (1808) Synonymia Insectorum, oder: versuch einer synonymie aller bisher bekannten Insecten, nach Fabricii Systema Eleutheratorum geordnet von C. J. Schönherr. Erster Band, Eleutherata oder Käfer. Zweiter Theil. C.F. Marquard, Stockholm, 424 pp.

Hoàng DN (1982) Coccinellidae of Vietnam Part I. Nha xuat ban khoa hoc va ky thuat, Hanoi, $211 \mathrm{pp}$.

Hoàng DN (1985) To the knowledge of ladybirds from Plateau Tay Nguyen, South Vietnam. Nauka, Moscow, 30-49. http://www.zin. ru/animalia/coleoptera/pdf/Hoàng85b.pdf

Huo LZ, Chen XS, Li WJ, Wang XM, Ren SX (2015) A new genus of the tribe Aspidimerini (Coleoptera Coccinellidae) from the Oriental Region. Annales Zoologici 65(2): 171-185. doi: 10.3161/00034541 ANZ2015.65.2.005

Huo LZ, Wang XM, Chen XS, Ren SX (2013) The genus Aspidimerus Mulsant, 1850 (Coleoptera, Coccinellidae) from China, with descriptions of two new species. ZooKeys 348: 47-75. doi: 10.3897/ zookeys.348.5746

Huo LZ, Wang XM, Chen XS, Ren SX (2014) The genus Pseudaspidimerus Kapur, 1948 (Coleoptera: Coccinellidae) from Laos, with descriptions of two new species. The Pan-Pacific Entomologist 90(4): 182-190. doi: 10.3956/2014-90.4.182

Kapur AP (1948) A revision of the tribe Aspidimerini Weise (Coleoptera, Coccinellidae). Transactions of the Royal Entomological Society of London 99: 77-128. doi: 10.1111/j.1365-2311.1948.tb01233.x

Kapur AP (1963) The Coccinellidae of the Third Mount Everest Expedition, 1924 (Coleoptera). Bulletin of the British Museum (Natural History) Entomology 14: 1-48.

Kovář I (2007) Coccinellidae. In: Löbl I, Smetana A (Eds) Catalogue of Palaearctic Coleoptera, Volume 4. Apollo Books, Stenstrup, 568-631.

Kuznetsov V, Pang H (1991) Description of Four New Aspidimerini (Coleoptera, Coccinellidae) from Vietnam. Elytra, Tokyo 19(2): 185-190.

Mader L (1933) Über bekannte und neue Coccinelliden. Entomologischen Anzeiger 5: 79-83. http://www.zobodat.at/pdf/EntAnz_13_0079-0084.pdf

Mader L (1954) Weiteres über Coccinelliden aus der Sammlung des Naturhistorischen Museums in Wien. Koleopterologische Rundschau, 32(1951-1954): 123-131.

Mulsant E (1850) Species des Coléoptères trimères sécuripalpes. Annales des Sciences Physiques et Naturelles, d'Agriculture et d'Industrie, Lyon (2) 2: 1-1104. doi: 10.5962/bhl.title.8953

Ohta Y (1929) Scymninen Japans. Insecta Matsumurana 4(1-2): 1-16.
Pang H (1998) The new record and new species of Aspidimerinae (Coleoptera: Coccinellidae) from China. Entomotaxonomia 20(3): 185-193.

Pang XF, Mao JL (1979) Economic Entomology of China 14: Coleoptera Coccinellidae II. Science Press, Beijing, 170 pp.

Poorani J (2002) An annotated checklist of the Coccinellidae (Coleoptera) (excluding Epilachninae) of the Indian subregion. Oriental Insects 36: 307-383. doi: 10.1080/00305316.2002.10417335

Ren SX, Wang XM, Pang H, Peng ZQ, Zeng T (2009) Colored pictorial handbook of ladybird beetles in China. Science Press, Beijing, 336 pp.

Sasaji H (1968) A revision of the Formosan Coccinellidae (II): Tribe Stethorini, Aspidimerini and Chilocorini (Coleoptera). Etizenia, Fukui (32): 1-24.

Ślipiński A (2007) Australian ladybird beetles (Coleoptera: Coccinellidae) their biology and classification. ABRS, Canberra, $286 \mathrm{pp}$.

Ślipiński A, Tomaszewska W (2010) Coccinellidae Latreille, 1802. In: Leschen RAB, Beutel RG, Lawrence JF (Eds) Handbook of Zoology, 2, Coleoptera. Walter de Gruyter GmbH \& Co KG, Berlin, New York, 454-472.

The Digital Museum of Natural \& Science: Cryptogonus kurosawai Sasaji, 1968. http://digimuse.nmns.edu.tw/Demo_2011/showMetadata. aspx OObjectId=0900000181d6eb28\& TypeKind=suMeta\&Type=type_minsect\&Part=2-2\&Domain=az\&Field=i1\&Language $=\mathrm{CHI}$

Weise J (1895) Deutsche Entomologische Zeitschrift, 1895: 326.

Weise J (1900) Coccinelliden aus Ceylon gesammelt von Dr. Horn. Deutsche Entomologische Zeitschrift 44: 417-448. doi: 10.1002/ mmnd.48019000237

Weise J (1910) Uber Chrysomeliden und Coccinelliden der Philippinen. Philippine Journal of Science 5D(4): 229-232.

Xiao NN, Li HX (1992) Coleoptera: Coccinellidae. In: Huang FS (Ed.) Insects of Wuling Mountains area, southwestern China. Science Press, Beijing, 368-390.

Yu GY (1995) The Coccinellidae (Excluding Epilachninae) collected by J. Klapperich in 1977 on Taiwan. Spixiana 18(2): 123-144. http:// biostor.org/reference/52566

Yu GY (2011) The Coccinellidae of Taiwan. Chemical Industry Press, Beijing, 198 pp.

Supplementary material 1

Specimens examined of Cryptogonus schraiki Mader, 1933

Authors: Lizhi Huo, Wenjing Li, Xiaosheng Chen, Shunxiang Ren, Xingmin Wang

Data type: Specimens examined.

Explanation note: 205 specimens of Cryptogonus schraiki Mader, 1933 were examined in the present study.

Copyright notice: This dataset is made available under the Open Database License (http://opendatacommons.org/licenses/ odbl/1.0/). The Open Database License (ODbL) is a license agreement intended to allow users to freely share, modify, and use this Dataset while maintaining this same freedom for others, provided that the original source and author(s) are credited. 